\title{
Corrigendum: The NCA sodium leak channel is required for persistent motor circuit activity that sustains locomotion
}

\author{
Shangbang Gao, Lin Xie, Taizo Kawano, Michelle D. Po, Jennifer K. Pirri, Sihui Guan, \\ Mark J. Alkema \& Mei Zhen
}

Nature Communications 6:6323 doi: 10.1038/ncomms7323 (2015); Published online 26 Feb 2015; Updated 21 May 2015

The authors inadvertently omitted Jennifer K. Pirri and Mark J. Alkema, who developed a promoter construct presented in this Article, from the author list, Acknowledgments, Author contributions statement and Methods section. In addition, in Fig. $3 \mathrm{~b}$, the $y$ axis was incorrectly labelled as Charge frequency (pC/s) instead of AP frequency $(\mathrm{Hz})$. In Fig. 4c the asterisks for significance were omitted from the third panel in the right. Finally, in Fig. 4e, Prgef-1 was incorrectly named promoter. All these errors have now been corrected in both the PDF and HTML versions of the Article. 